\title{
Innovación tecnológica para recuperar el algodón nativo de color ${ }^{1}$
}

\author{
Dora Cortijo de Arbildo \\ Universidad de Lima. Lima, Perú \\ Correo electrónico: Dcortijo@ulima.edu.pe \\ Rómulo Saúl Cancio Iparraguirre \\ Universidad de Lima. Lima, Perú \\ Correo electrónico: Rcancio@correo.ulima.edu.pe
}

Recibido: 11/5/2012 / Aprobado: 8/7/2012

\begin{abstract}
Resumen: Se busca adaptar la tecnología existente para el algodón comercial al hilado de algodón nativo de color (ANC), que, por su fibra corta, no puede ser procesado industrialmente con la tecnología existente. Así, se ensayó mezclando ANC puro con algodón Tangüis para determinar sus características físicas, como elongación, resistencia y finura de la fibra. Se encontró que una mezcla de $63 \%$ de ANC y $37 \%$ de algodón Tangüis permite obtener un hilo de características adecuadas para tejer prendas de vestir.
\end{abstract}

Palabras clave: Algodón / algodón nativo de color / tejidos de algodón / tecnología textil

\section{Technological innovation to retrieve the peruvian native color cotton (ANC)}

ABSTRACT: Seeks to adapt the current technology of commercial cotton to the knitting of Natural Peruvian Color Cotton (ANC), it cannot be industrially processed with the current technology because of its short fiber. There has been developed trials with pure ANC mixed with Tangüis cotton, in order to determine their physical attributes such as length, resistance and fiber thinness, finding that $63 \%$ of ANC mixed with $37 \%$ of Tangüis cotton makes possible to obtain a string with adequate characteristics to make clothing pieces.

Keywords: Cotton / native color cotton / cotton knitting / textile technology

1 Este trabajo forma parte de la investigación "Innovación tecnológica para recuperar el algodón nativo de color" que han desarrollado los autores con el patro- 


\section{INTRODUCCIÓN}

En las épocas preínca e inca se utilizó extensivamente el ANC, tanto para la elaboración de prendas de vestir y accesorios (Vreeland Jr., 1999) como para fines medicinales. Actualmente se hila y se teje ANC de manera artesanal con muy baja productividad, obteniéndose hilo grueso apto para elaborar carteras, adornos y otros accesorios. Se sabe que en el mercado norteamericano existen algunas empresas que ofrecen prendas de vestir confeccionadas con ANC comprado a las artesanas de Mórrope y a agricultores del departamento de San Martín; sin embargo, en nuestro país no existe la tecnología para ofrecer este tipo de prendas.

Con esta información se buscó alternativas tecnológicas para su procesamiento industrial y la obtención de tela de punto de calidad adecuada para la fabricación de prendas de vestir, encontrándose que otras entidades del país han desarrollado o están desarrollando trabajos en la misma dirección, como el Senati, Cáritas del Perú, AgroRural, entre otras.

\section{DESARROLLO DEL PROYECTO}

\subsection{Diagnóstico}

El algodón es un recurso natural que se presenta en diferentes variedades: Pima, Tangüis, del Cerro y Áspero. En el Perú antiguo la variedad que más se empleó fue el Áspero; al respecto, se ha encontrado algodón de color en los restos de la cultura precolombina, al que llamaremos algodón nativo de color (ANC).

Actualmente se conoce poco acerca del ANC y su existencia ha sido muy poco difundida, tanto en el mercado nacional como en el internacional, a pesar de las bondades que presenta este tipo de fibra. Entre ellas destacan el hecho de que no requiere ningún tipo de teñido, ya que sus colores son naturales, lo cual hace que las personas alérgicas o de piel sensible a los tintes químicos puedan utilizar prendas hechas

cinio del Instituto de Investigación Científica de la Universidad de Lima, con la participación de las exalumnas Paloma Torres en el diseño de encuestas y trabajo de campo, Verónica Michue en trabajo de campo y el alumno Rubén Manrique en el diseño de las máquinas. 
sobre la base de este tipo de fibra. Además tiene un gran potencial para la industria de confección, con respecto a otros algodones comerciales como el Tangüis o el Pima.

En el Perú, el cultivo de ANC se centra principalmente en la zona norte, sobre todo en los departamentos de Lambayeque, Piura y San Martín. En Lambayeque, el cultivo de ANC se encuentra mayoritariamente en los distritos de San José, Túcume, Mórrope y en la provincia de Ferreñafe. Actualmente, el Ministerio de Agricultura, a través del Programa de Desarrollo Productivo Agrario Rural (AgroRural), está realizando trabajos de cultivo de esta planta, y ya tiene sembrados más de 20.000 plantones de ANC en la zona de Picsi, distrito de la provincia de Chiclayo.

En el distrito de Mórrope se observó la forma artesanal como las campesinas siembran y procesan el algodón nativo de color. Aquí se encontró que ellas preservan un conocimiento ancestral heredado de sus antepasados y transmitidos de madres a hijas. En las entrevistas realizadas para el presente estudio, manifestaron un alto interés en trabajar con algún tipo de máquina que las ayude a mejorar su productividad y calidad del algodón. Actualmente, los ingresos que perciben por sus productos (carteras, bolsos, billeteras, portacelulares, etcétera) no les permiten sustentarse económicamente y, por eso, no muestran mayor dedicación al cultivo del algodón nativo.

El proceso artesanal del algodón nativo de color (ANC) se compone de los siguientes pasos:

- Despepitado.- Consiste en el retiro de las semillas de las bellotas. Aproximadamente se pueden obtener de 6 a 8 semillas por capullo.

- Vareado.- Después de que los capullos han quedado limpios, las artesanas juntan aproximadamente 5 kilos de ANC, que pasan a ser golpeados con dos varas de madera, una en cada mano, aproximadamente por un periodo de 30 minutos hasta obtener una alfombrilla de algodón conocido como 'napa', cuyas dimensiones varían entre 50 y 60 centímetros de ancho y de 80 a 90 centímetros de largo, aproximadamente. Esta operación se puede observar en la foto $1 .{ }^{2}$

2 Tanto las fotos como los cuadros y las figuras que ilustran el presente artículo, han sido proporcionados por el Centro de Estudios Ambientales (CEA) de la Universidad de Lima. 
Foto 1

Vareado de la mezcla ANC y Tangüis

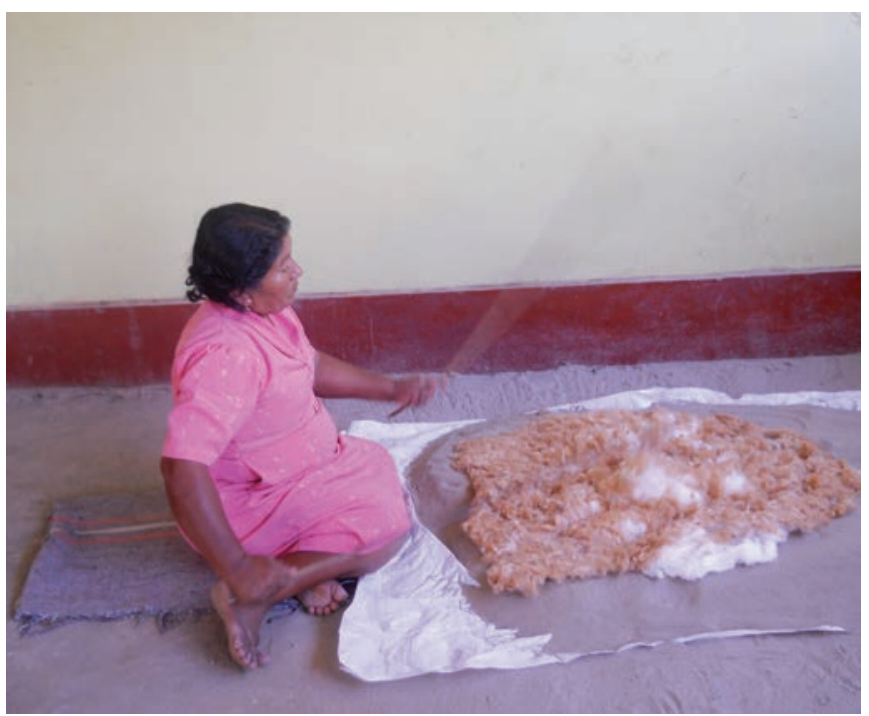

- Formación de copos.- Con la napa obtenida de la operación anterior, se procede a la formación del copo, el cual se consigue doblando la napa en dos y luego enrollándola. Se obtiene un rollo rectangular, tal como se muestra en las fotos 2 y 3.

Foto 2

Acomodando la mezcla para formar el rollo

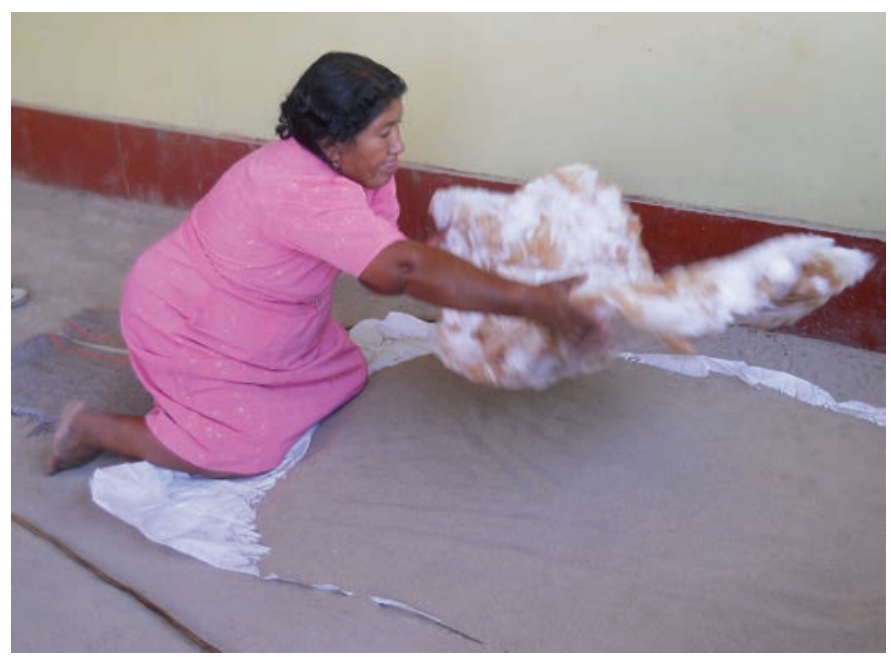


Foto 3

Copos (rollos) de algodón nativo de color

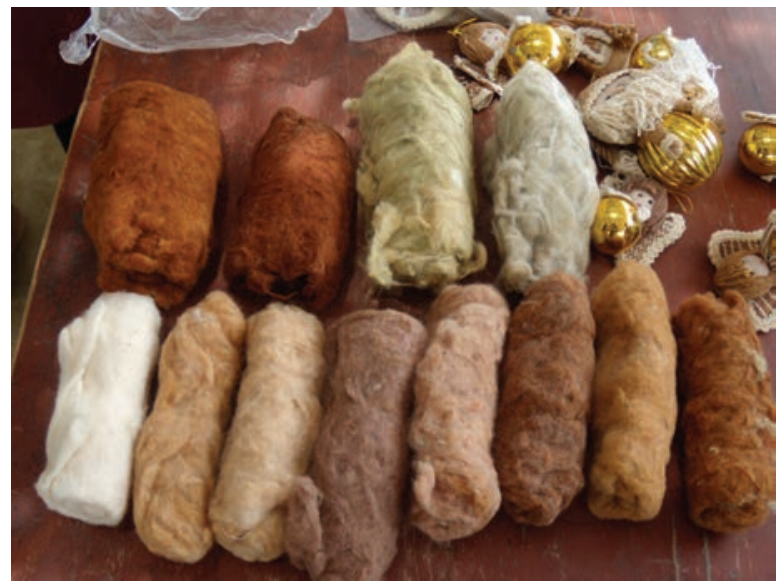

- Hilado.- El copo se amarra a una estaca o tronco; las artesanas desprenden con los dedos índice y medio de la mano izquierda una porción de $\mathrm{ANC}$, otorgándole al mismo tiempo una ligera torsión, mientras que con la mano derecha lo jalan o estiran para formar una mecha, creando de esta manera la operación de estiraje. Este hilo obtenido es recolectado en un huso en el cual lo va enrollando. Esta operación se muestra en la foto 4 .

\section{Foto 4}

Hilando la mezcla de ANC y Tangüis

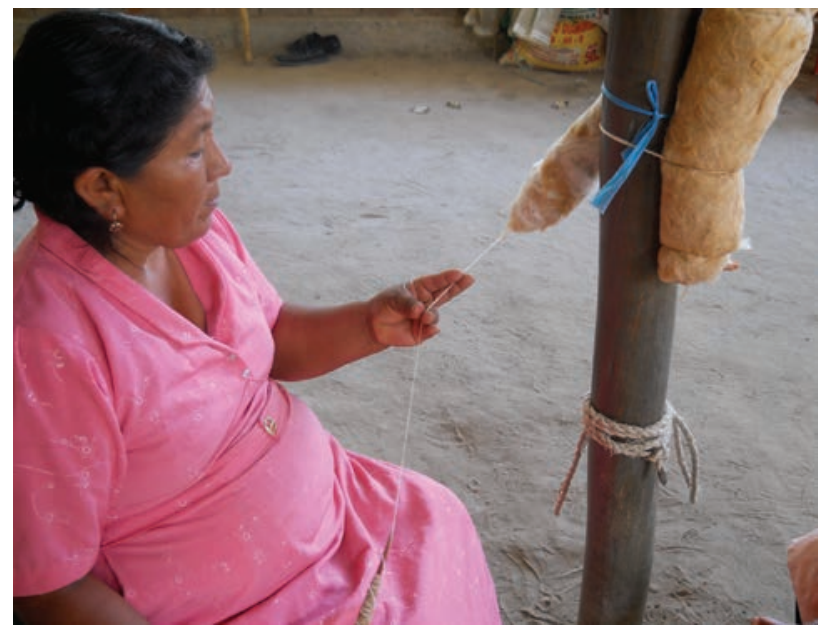


- Ovillado.- Luego de obtener el hilo se pasa al ovillado, para el cual son necesarios aproximadamente de 3 a 4 husos, de los cuales se obtiene un ovillo, tal como se observa en la foto 5.

Foto 5

Ovillos de hilo de ANC y Tangüis mezclados y de ANC puro

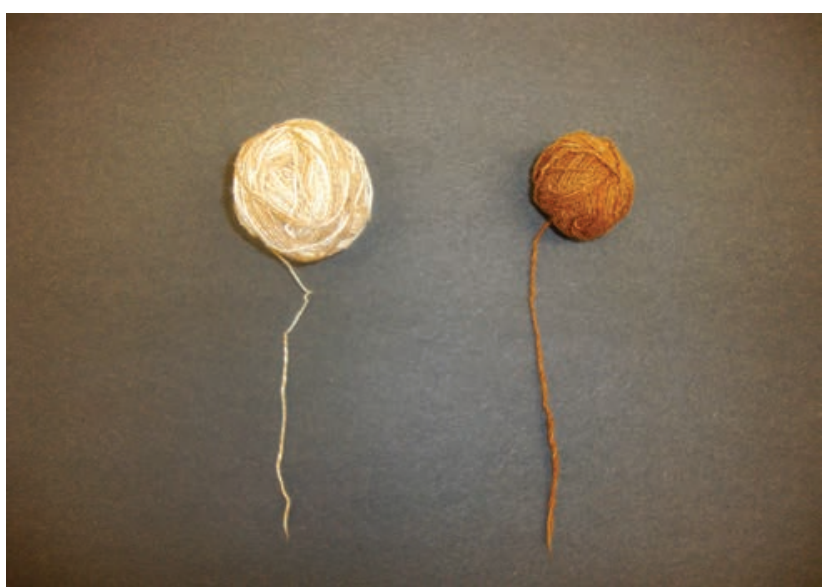

- Urdido.- Consiste en el ordenamiento del hilo de forma paralela en estacas o palos distribuidos en forma geométrica (cuadrado o rectángulo). La longitud de la urdiembre ${ }^{3}$ dependerá mucho de las dimensiones que se quiere para la tela que se utilizará en la etapa de confección.

- Tejido. - Tal como se muestra en la foto 6, las artesanas trabajan con telares de cintura, en los que la urdiembre será tensada con el telar, sujetándola de un extremo a un soporte vertical (palo) y los otros extremos se colocan en la parte posterior de la cintura, a manera de una faja (emuque).

Todo el proceso seguido por las artesanas se asemeja de manera simplificada a un proceso industrial de hilado de algodón (Red Textil Argentina, 2008).

3 Conjunto de hilos que se colocan paralelamente unos a otros para formar una tela. 
Foto 6

Tejiendo ANC

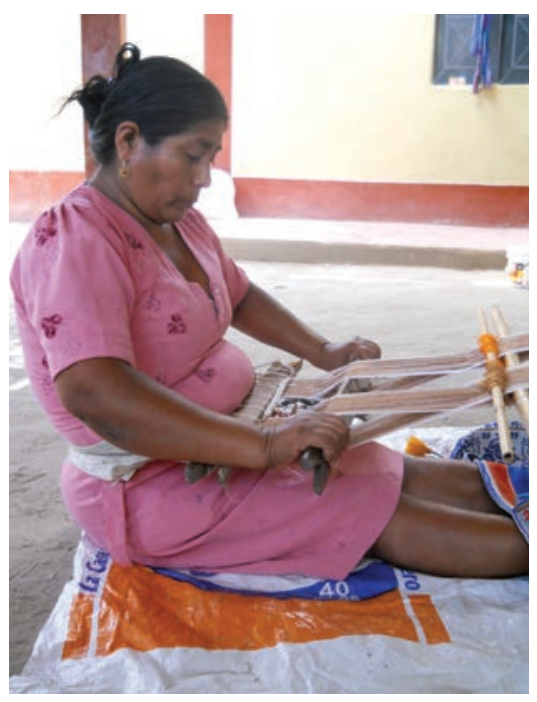

- Confección.- En esta operación las artesanas aplican su creatividad para elaborar carteras, bolsos, mantas, billeteras, adornos, etcétera.

Foto 7

Artículos artesanales de ANC

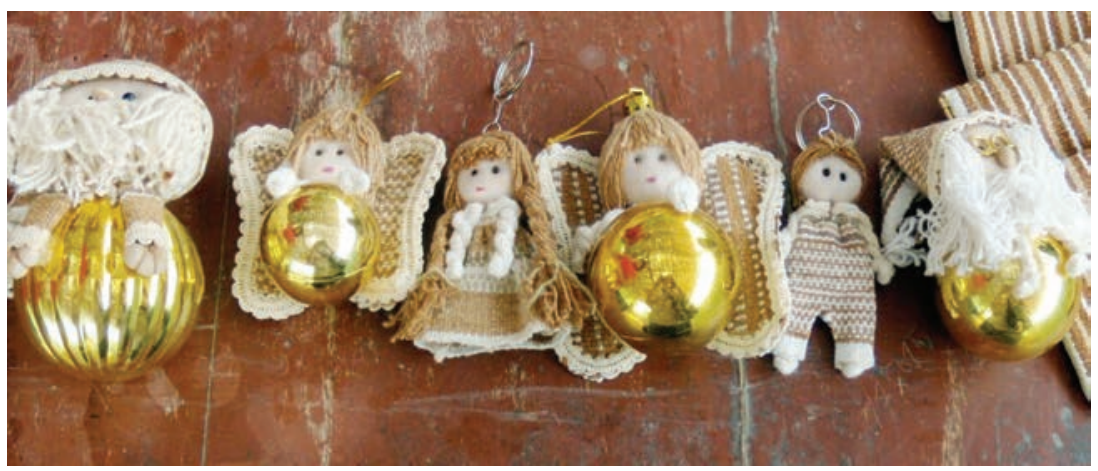

\section{ANÁLISIS DEL ANC Y DE HILO FABRICADO CON ANC}

Se procedió a analizar tanto el ANC como los hilos de ANC fabricados por las artesanas de Mórrope, comparándolos con los valores encontrados para el algodón Tangüis y el hilo fabricado con este por las artesanas. Se encontró que la fibra del ANC es más corta y su resistencia es 
menor; asimismo, tiene menos finura que la fibra de algodón Tangüis. Se analizaron las características del hilo (DeTextiles, 2009), que facilitan su uso para el tejido de prendas.

\subsection{Resultados del análisis de la fibra de $\mathrm{ANC}^{4}$}

Como se señaló, las fibras del ANC y del Tangüis poseen características diferentes, tal como se observa en el cuadro 1, mientras que en el cuadro 2 se puede apreciar que en los hilos obtenidos mediante hilatura manual (artesanal) los valores de torsión, resistencia y título dependen sobre todo de la experticia de la artesana que hila el ANC.

Cuadro 1

Resultados de los análisis de fibra ANC

\begin{tabular}{lcc}
\hline Análisis & ANC & Tangüis \\
\hline Longitud de fibra (mm) & 24.13 & 33.02 \\
Resistencia (lbs./pul2) & 88.21 & 93.65 \\
Finura (micronaire) & 6.2 & 4.7 \\
\hline
\end{tabular}

Cuadro 2

Análisis de hilo artesanal de ANC

\begin{tabular}{|l|c|c|c|c|}
\hline \multicolumn{2}{|c|}{$\begin{array}{c}\text { Algodón } \\
\text { áspero } \\
\text { Muestra 1 }\end{array}$} & $\begin{array}{c}\text { Algodón } \\
\text { áspero } \\
\text { Muestra 2 }\end{array}$ & $\begin{array}{c}\text { Algodón } \\
\text { áspero } \\
\text { Muestra 3 }\end{array}$ & $\begin{array}{c}\text { Algodón } \\
\text { áspero } \\
\text { Muestra 4 }\end{array}$ \\
\hline \multicolumn{5}{|c|}{ Título } \\
\hline Ne promedio real & 4.91 & 4.68 & 4.98 & 5.6 \\
\hline Ne teórico & $5 / 1$ & $5 / 1$ & $7 / 1$ & $6 / 1$ \\
\hline \multicolumn{5}{|c|}{ Torsiones } \\
\hline Promedio TPM & 640 & 545 & 475.8 & 537.6 \\
\hline Promedio TPP & 16.26 & 13.84 & 12.09 & 13.66 \\
\hline Alfa de torsión & 7.34 & 6.40 & 4.57 & 5.77 \\
\hline
\end{tabular}

(continúa)

4 Los diferentes análisis fueron hechos en las empresas Coats Cadena S. A., Textiles del Sur S. A. C., Certintex S. A. C. y Quality Laba S. A. C. 
(continuación)

\begin{tabular}{|c|c|c|c|c|}
\hline & $\begin{array}{l}\text { Algodón } \\
\text { áspero } \\
\text { Muestra } 1\end{array}$ & $\begin{array}{c}\text { Algodón } \\
\text { áspero } \\
\text { Muestra } 2\end{array}$ & $\begin{array}{c}\text { Algodón } \\
\text { áspero } \\
\text { Muestra } 3\end{array}$ & $\begin{array}{c}\text { Algodón } \\
\text { áspero } \\
\text { Muestra } 4\end{array}$ \\
\hline \multicolumn{5}{|c|}{ Resistencia } \\
\hline $\begin{array}{l}\text { Resistencia } \\
\text { mínima - cN }\end{array}$ & 437.48 & 619.76 & 382.79 & 537.6 \\
\hline $\begin{array}{l}\text { Resistencia } \\
\text { máxima - cN }\end{array}$ & 637.99 & 1057.2 & 583.3 & 1020.8 \\
\hline $\begin{array}{l}\text { Resistencia } \\
\text { promedio - cN }\end{array}$ & 495.81 & 809.93 & 488.52 & 1604.1 \\
\hline $\begin{array}{l}\text { Resistencia } \\
\text { promedio - g }\end{array}$ & 505.57 & 825.87 & 498.13 & 1305.1 \\
\hline CV\% Resistencia & 17.13 & 13.49 & 16.56 & 1330.8 \\
\hline Tenacidad - Cn/tex & 4.13 & 6.42 & 5.78 & 18.68 \\
\hline Tenacidad - RKM & 4.21 & 6.55 & 5.89 & 12.40 \\
\hline & & & & 8 \\
\hline
\end{tabular}

Elaboración propia.

En cuanto al análisis del hilado de ANC con algodón blanco Tangüis (ABC) que se aprecia en los cuadros 3 y 4, en diferentes proporciones de mezcla, tanto a nivel artesanal como semiindustrial, este se realizó en la planta piloto de la empresa Textiles del Sur, ${ }^{5}$ en Chincha. Como se observa, existe una alta variabilidad en los resultados del análisis de las muestras hiladas artesanalmente.

5 La empresa quebró a fines del año 2010, por lo que no se pudo seguir con las pruebas piloto. 
Cuadro 3

Características de hilos artesanales de ANC y de algodón Tangüis

\begin{tabular}{lcccc}
\hline \multirow{2}{*}{ Análisis } & \multicolumn{2}{c}{ ANC $\mathbf{1 0 0 \%}$} & \multicolumn{2}{c}{ Tangüis $\mathbf{1 0 0 \%}$} \\
\hline & Hilatura normal & Hilatura fina & Hilatura normal & Hilatura fina \\
\hline Resistencia $(\mathrm{N})$ & 600.0 & 566.1 & 683.5 & $*$ \\
Elongación (\%) & 5.6 & 4.6 & 2.9 & 6.24 \\
Título (Ne) & 3.95 & 4.60 & 4 & 5 \\
\hline
\end{tabular}

Elaboración propia

Cuadro 4

Características de hilos artesanales obtenidos a partir de ANC y algodón Tangüis mezclados en diferentes proporciones

\begin{tabular}{|c|c|c|c|c|c|c|}
\hline \multirow[t]{2}{*}{ Análisis } & \multicolumn{2}{|c|}{$\begin{array}{l}\text { Porcentaje de mezcla: } \\
75 \text { ANC / } 25 \text { Tangüis }\end{array}$} & \multicolumn{2}{|c|}{$\begin{array}{l}\text { Porcentaje de mezcla: } \\
50 \text { ANC I } 50 \text { Tangüis }\end{array}$} & \multicolumn{2}{|c|}{$\begin{array}{l}\text { Porcentaje de mezcla: } \\
25 \text { ANC I } 75 \text { Tangüis }\end{array}$} \\
\hline & $\begin{array}{c}\text { Hilatura } \\
\text { normal }\end{array}$ & $\begin{array}{l}\text { Hilatura } \\
\text { fina }\end{array}$ & $\begin{array}{l}\text { Hilatura } \\
\text { fina }\end{array}$ & $\begin{array}{l}\text { Hilatura } \\
\text { normal }\end{array}$ & $\begin{array}{l}\text { Hilatura } \\
\text { fina }\end{array}$ & $\begin{array}{l}\text { Hilatura } \\
\text { normal }\end{array}$ \\
\hline Resistencia (N) & 615.4 & 838.2 & 838.2 & 582.6 & * & 583.1 \\
\hline Elongación (\%) & 11.6 & 8.2 & 8.2 & 11.6 & 3.0 & 10.1 \\
\hline Título (Ne) & 4.25 & 4.07 & 4.07 & 4.25 & 3.48 & 4.25 \\
\hline
\end{tabular}

De lo analizado, se observa que en la mezcla de $75 \%$ de ANC y $25 \%$ de algodón Tangüis el hilo presenta la mejor calidad en cuanto a resistencia y elongación en hilatura normal, pero, mediante una proyección matemática, se encuentra que la mejor calidad de hilo se obtendría con una mezcla de $63 \%$ ANC y $37 \%$ de algodón Tangüis en hilatura fina.

Para otra prueba se adquirió ANC hilado industrialmente con una mezcla de $60 \%$ de ANC y $40 \%$ de Tangüis, elaborado por un proyecto de Cáritas del Perú, ${ }^{6}$ cuyos valores, luego del análisis, se muestran en el cuadro 5. En este se observa que el hilo de algodón nativo tejido de manera industrial tiene mejores título (más fino) y torsión, pero su resistencia es menor en comparación con los hilos artesanales.

6 Cáritas del Perú viene desarrollando un proyecto con la comunidad del bosque de Pómac, en Ferreñafe, Lambayeque, con el objetivo de fortalecer el cultivo y la industrialización de ANC. 


\section{Cuadro 5}

Valores de análisis de hilo comprado a Cáritas del Perú

\begin{tabular}{lccc}
\hline & \multicolumn{3}{c}{ Tipo de hilatura } \\
\cline { 2 - 4 } & $\begin{array}{c}\text { Industrial } \\
\text { (60\% ANC/40\% ABC) }\end{array}$ & $\begin{array}{c}\text { Artesanal } \\
\text { (60\% ANC/40\% ABC) }\end{array}$ & $\begin{array}{c}\text { Artesanal } \\
\text { (100\% ANC) }\end{array}$ \\
\hline Torsión (TPP) & 23.45 & 11.6 & 12.09 \\
Resistencia (cN) & 356 & 1241 & 488.52 \\
Titulo (Ne) & 12.9 & 3.4 & 7 \\
\hline
\end{tabular}

Elaboración propia.

El mejoramiento de las variables se puede conseguir con las mezclas; por ejemplo, un mejor título y torsión permitirán obtener hilos para telas (tejido plano o de punto) con las cuales se pueden confeccionar prendas de vestir de diferentes texturas ${ }^{7}$ semiindustriales.

\subsection{Análisis de la tela fabricada con algodón nativo con un porcentaje de mezcla de $60 \%$ de ANC y $40 \%$ de Tangüis}

A partir del hilo comprado a Cáritas del Perú, se tejieron dos muestras de tela en tejido jersey ${ }^{8}$ de 1,20 metros por 0,45 centímetros, cada una en una máquina semiindustrial, con número de galga 6 (cantidad de agujas por pulgada), una de ellas tejida con hilo de un cabo y la otra con dos. Estas arrojaron los resultados mostrados en el cuadro 6 y la figura 1.

7 Diferentes tejidos de tela con variación del número de cabos y tipo de tejido.

8 Presenta una superficie plana y un revés caracterizado por la aparición de bucles cortos dispuestos horizontalmente. 


\section{Cuadro 6}

Análisis de muestras de tela (tejido jersey) de ANC

\begin{tabular}{|c|c|c|c|c|c|c|}
\hline & Características & \multicolumn{2}{|c|}{$\begin{array}{l}\text { Muestra A } \\
\text { (un cabo) }\end{array}$} & \multicolumn{2}{|c|}{$\begin{array}{l}\text { Muestra B } \\
\text { (dos cabos) }\end{array}$} & $\begin{array}{c}\text { Valor } \\
\text { recomendable }\end{array}$ \\
\hline (1) & \multirow{2}{*}{$\begin{array}{l}\text { Resistencia al desli- } \\
\text { zamiento de los hilos } \\
\text { en la costura (lbf.) }\end{array}$} & Vertical & Horizontal & Vertical & Horizontal & \\
\hline (2) & & 13.2 & 7.6 & 21.2 & 25.4 & 23 \\
\hline (3) & $\begin{array}{l}\text { Apariencia después } \\
\text { del lavado (SA) }\end{array}$ & \multicolumn{2}{|c|}{$\begin{array}{c}3.33 \\
\text { Apariencia algo arrugada } \\
\text { (poco perceptible) }\end{array}$} & \multicolumn{2}{|c|}{$\begin{array}{c}3.17 \\
\text { Apariencia algo arruga- } \\
\text { da (poco perceptible) }\end{array}$} & 5 \\
\hline (4) & $\begin{array}{l}\text { Resistencia al estalli- } \\
\text { do hidráulico (PSI) }\end{array}$ & \multicolumn{2}{|c|}{43.1} & \multicolumn{2}{|c|}{73.4} & > $55 \mathrm{PSI}$ \\
\hline (5) & $\begin{array}{l}\text { Resistencia a la for- } \\
\text { mación de pilosidad }\end{array}$ & \multicolumn{2}{|c|}{$\begin{array}{c}3-4 \\
\text { Ligero a moderado } \\
\text { Pillimg }\end{array}$} & \multicolumn{2}{|c|}{$\begin{array}{c}3-4 \\
\text { Ligero a moderado } \\
\text { Pillimg }\end{array}$} & 5 \\
\hline \multirow[t]{2}{*}{ (7) } & \multirow{2}{*}{$\begin{array}{l}\text { Solidez del color al } \\
\text { frote }\end{array}$} & $\begin{array}{l}\text { Grado } \\
\text { seco }\end{array}$ & $\begin{array}{l}\text { Grado } \\
\text { húmedo }\end{array}$ & $\begin{array}{l}\text { Grado } \\
\text { seco }\end{array}$ & $\begin{array}{l}\text { Grado } \\
\text { húmedo }\end{array}$ & \multirow[t]{2}{*}{5} \\
\hline & & 5 & 4.5 & 5 & 4.5 & \\
\hline (8) & $\begin{array}{l}\text { Solidez del color a la } \\
\text { transpiración }\end{array}$ & & 4 & & .5 & 4 \\
\hline
\end{tabular}

Figura 1

Muestras de tela

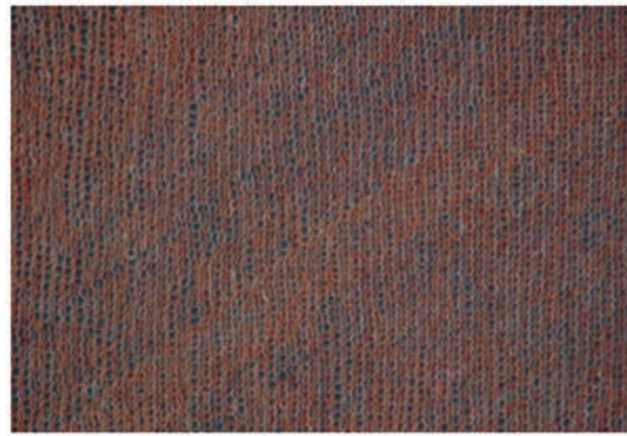

Muestra A: T2.1 (1 cabo)

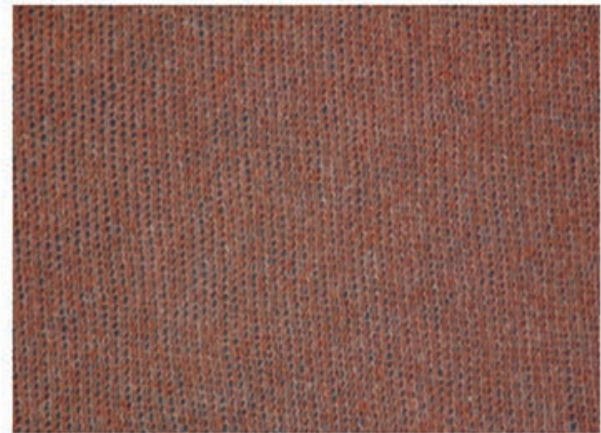

Muestra B: T2.1 (dos cabos) 
De acuerdo con los análisis realizados a las muestras de hilo, se pudo observar que el algodón fue hilado con demasiada torsión, ${ }^{9}$ lo que dificultó el tejido de las muestras en una máquina de tejer semiindustrial rectilínea. Así, se puede concluir que para lograr prendas con los debidos estándares de calidad, en la etapa de hilado industrial se deben controlar las variables de torsión y resistencia. En cuanto a la textura, es decir el número de cabos (hilo) usados para tejer las muestras de tela de ANC, esta es importante, pues cuanto más cabos tenga la tela, tendrá también más resistencia para soportar los diferentes tratos a que será sometida.

\section{DISEÑO CONCEPTUAL DE DESMOTADORA}

La operación de despepitar el algodón, es decir de retirarle las semillas, es fundamental, y aunque aún se realiza manualmente en diversos valles, alcanzando a despepitar 1 kilo de algodón en 6 horas, existen las máquinas desmotadoras (figura 2), que facilitan esta labor, pues la realizan de forma mecánica.

Las dimensiones que se proponen en nuestro estudio son: 0,50 centímetros de ancho, 110 centímetros de alto y 0,75 centímetros de largo; la alimentación de la materia prima se realizará por la parte superior del módulo, que trabaja con tres rodillos cuyo movimiento es accionado por una manivela que la hará girar y, mediante engranajes, se transmitirá el movimiento a todo el sistema.

Cada rodillo tiene una característica distinta: el primer rodillo presenta dientes distribuidos en 6 secciones a lo largo de su superficie, los cuales permiten arrastrar la materia prima hacia el siguiente rodillo, que se encargará de retirar las semillas. Este rodillo presenta pequeños ganchos (dientes) a lo largo de su superficie.

El tercer rodillo permitirá retirar la fibra, sin semillas, para lo cual se ha dispuesto de 6 juegos de escobillas a lo largo de la superficie del rodillo. Las semillas serán almacenadas en una bandeja que se encuentra dispuesta en la parte posterior del módulo.

9 Número de vueltas en un metro de hilo. 
Figura 2

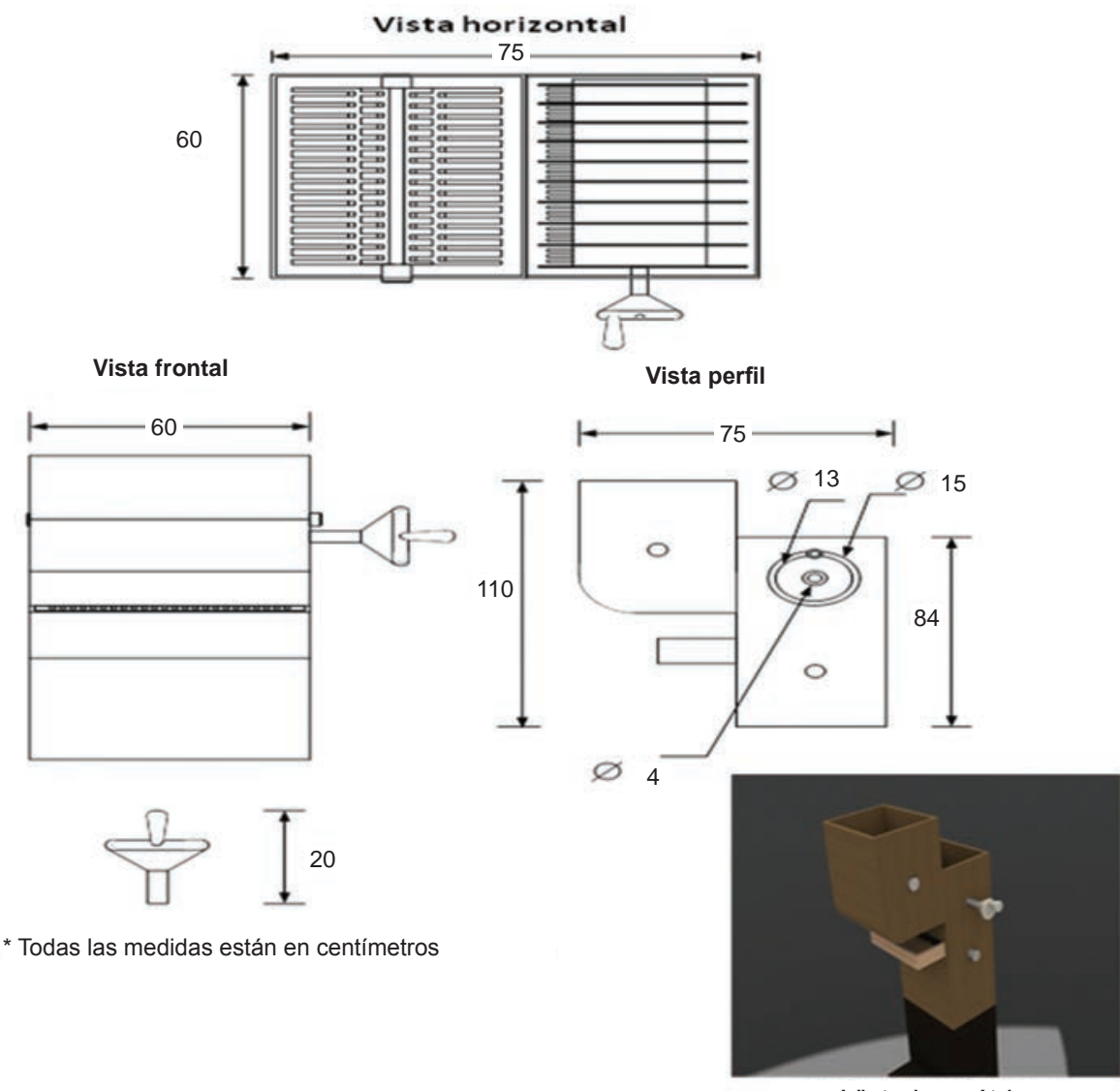

Vista isométrica

\section{DISEÑO CONCEPTUAL DE CARDA}

La función de esta máquina es uniformizar y paralelizar las fibras de algodón hasta obtener un velo de fibras casi transparente, lo cual se logra mediante un sistema de rodillos que se encontrará dispuesto de tal forma que permita el acceso y la salida de la materia prima. En la figura 2 se observa que el rodillo grande de 10 centímetros de diámetro está compuesto por pequeños dientes, los cuales permiten realizar el peinado ${ }^{10}$ de la fibra, así como también la eliminación de las fibras más cortas y de algunos elementos extraños (pajas, semillas, etcétera).

10 Consiste en paralelizar las fibras, lo cual permitirá efectuar un buen proceso de hilado. 
La recepción del velo obtenido será de forma continua. Para esto se ha acondicionado otro rodillo con agujas muy delgadas y, a través de movimientos de subida y bajada, permitirá el retiro del velo del algodón formado. Este mecanismo tiene su propia fuente de energía, generada por un motor.

El funcionamiento de este módulo será manual. El uso de una manivela accionará el sistema y, mediante el juego de poleas, se transmitirá el movimiento a los rodillos.

El objetivo de este módulo es mejorar el proceso de vareado que las artesanas desarrollan actualmente, reducir el tiempo de operación, así como obtener un mejor paralelismo de las fibras, lo cual permitirá obtener un hilo de calidad.

Figura 3

Vistas de la carda*

Vista frontal

*Todas las medidas están en centímetros. 


\section{DISEÑO CONCEPTUAL DE MÁQUINA SEMIINDUSTRIAL PARA HILAR}

\subsection{Funcionamiento interno de la máquina}

Las máquinas hilanderas cumplen la función de hilar el algodón, existen máquinas industriales que pueden llegar a trabajar con más de cien husos en simultáneo. En el caso de nuestro proyecto se buscará la adaptación del funcionamiento de estas máquinas industriales para el diseño de una máquina hilandera semiindustrial de 4 husos.

En la figura 6 se muestra el diseño semiindustrial desarrollado con un motor de $3 \mathrm{HP}$, el que se encargará de dar potencia a todas las partes móviles de la máquina a través de un sistema de poleas. El motor le transfiere potencia de manera directa a un primer eje a través de una faja.

Para la elaboración del diseño se ha tomado como base la máquina continua de hilar por anillos, que la Universidad Nacional de Ingeniería tiene en su laboratorio de textiles con un motor de 1HP, $220 \mathrm{~V}$. Sin embargo, para facilitar su manipulación y su mantenimiento se buscó cambiar tanto el mecanismo interno como los medios de manipulación. A continuación se detallan los cambios realizados y se explica cómo estos pueden facilitar el trabajo de los operarios.

\subsection{Alternativas de funcionamiento}

Se podrían definir varias alternativas de funcionamiento:

- Bancada.- Para realizar el movimiento de subida y de bajada, originalmente se empleaba lo que se llamaba una caja de cambios, la cual consistía en un juego de engranajes y un gatillo.

En el caso de nuestra máquina, se empleará un "controlador de velocidad", el cual solo se encargará de disminuir la velocidad de giro que llega a la bancada. Después se une a dos engranajes que, juntos, dan el movimiento de subida y de bajada a la bancada, como se muestra en la figura 4. 
- Control de velocidad.- Para el control de la velocidad, en la máquina original se utiliza una serie de engranajes de diferentes tamaños controlados por una cadena, la cual va rotando de engranajes mediante un timón ubicado en la parte frontal de la máquina, tal como se ve en la figura 4.

Con la máquina que se ha diseñado, se busca evitar la complejidad de un sistema de cambio de velocidad basado en engranes y en reemplazo un motor cuya potencia y velocidad de giro se pueda controlar mediante la aplicación de una determinada fuerza.

Para esto se ideó un sistema de presión que funciona girando un timón ubicado en la parte frontal de la máquina. Este hace girar dos engranes, que le bajan fuerza a una palanca que se encarga de mover el motor. De esta manera, los operarios pueden controlar la velocidad de giro de los usos y de la bancada.

- Independencia del tren de estiraje.- El estiraje es una operación que permite agrupar las fibras en forma paralela y uniforme para obtener un hilo continuo (Ingeniería textil, 2009).

En la máquina original se emplea un motor de $3 \mathrm{HP}$ para mover todos los mecanismos de la máquina.

Para este caso se propone independizar ciertas áreas de la máquina, de modo que se puedan utilizar motores de menor potencia y por ende ahorrar en costos. Además, al independizar las partes se logra eliminar los complejos sistemas de poleas y ejes que sirven para comunicar todos los mecanismos con el motor.

$\mathrm{Al}$ respecto, se propone usar un motor de 300 watts para mover la bancada y los 4 husos. Este motor contará con el sistema de control de velocidad mostrado en la figura 5 .

Finalmente, se independizará el tren de estiraje mediante la implementación de 3 motores de 50 watts cada uno, que moverá uno de los tres ejes en el tren. Para sincronizar el movimiento de estos tres ejes se conectarán los tres motores a un solo interruptor, de modo que enciendan al mismo tiempo. 
Figura 4

Mecanismo de movimiento control de la velocidad (frontal)
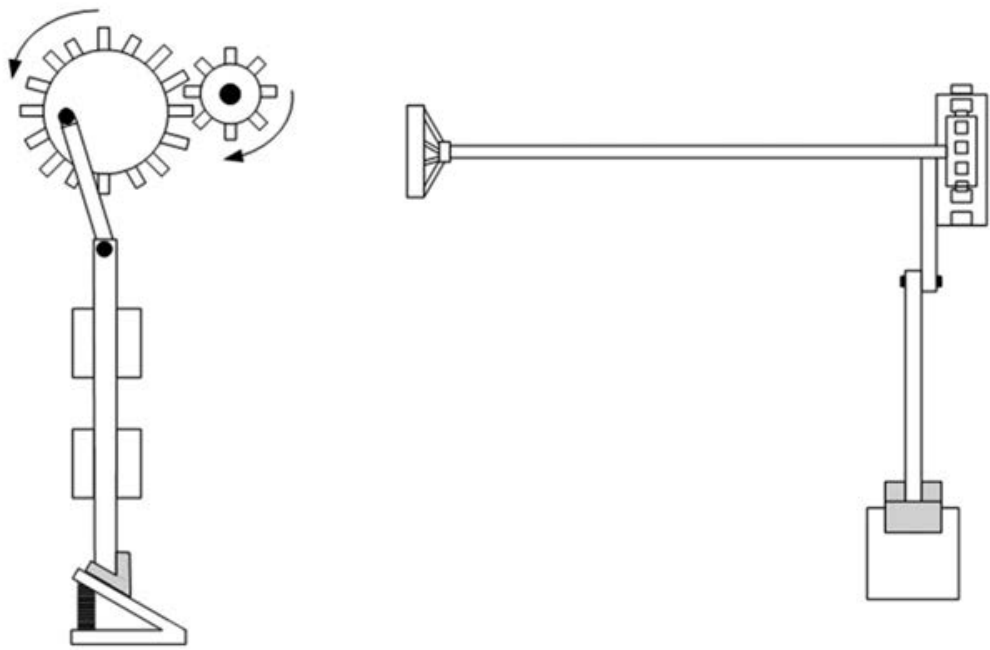

Figura 5

Tren de estiraje propuesto

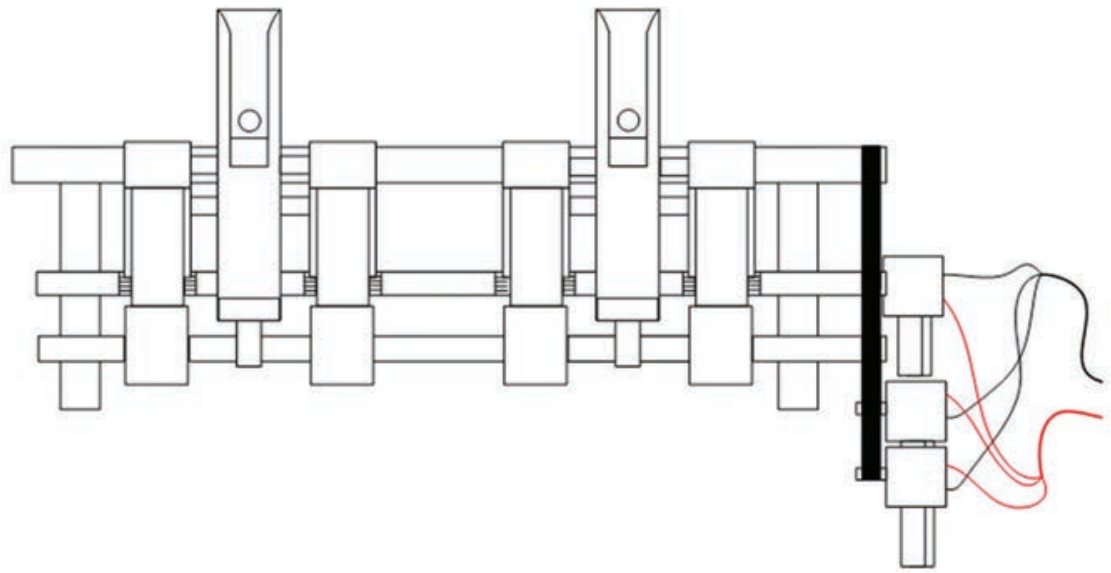


Figura 6

Vistas del diseño

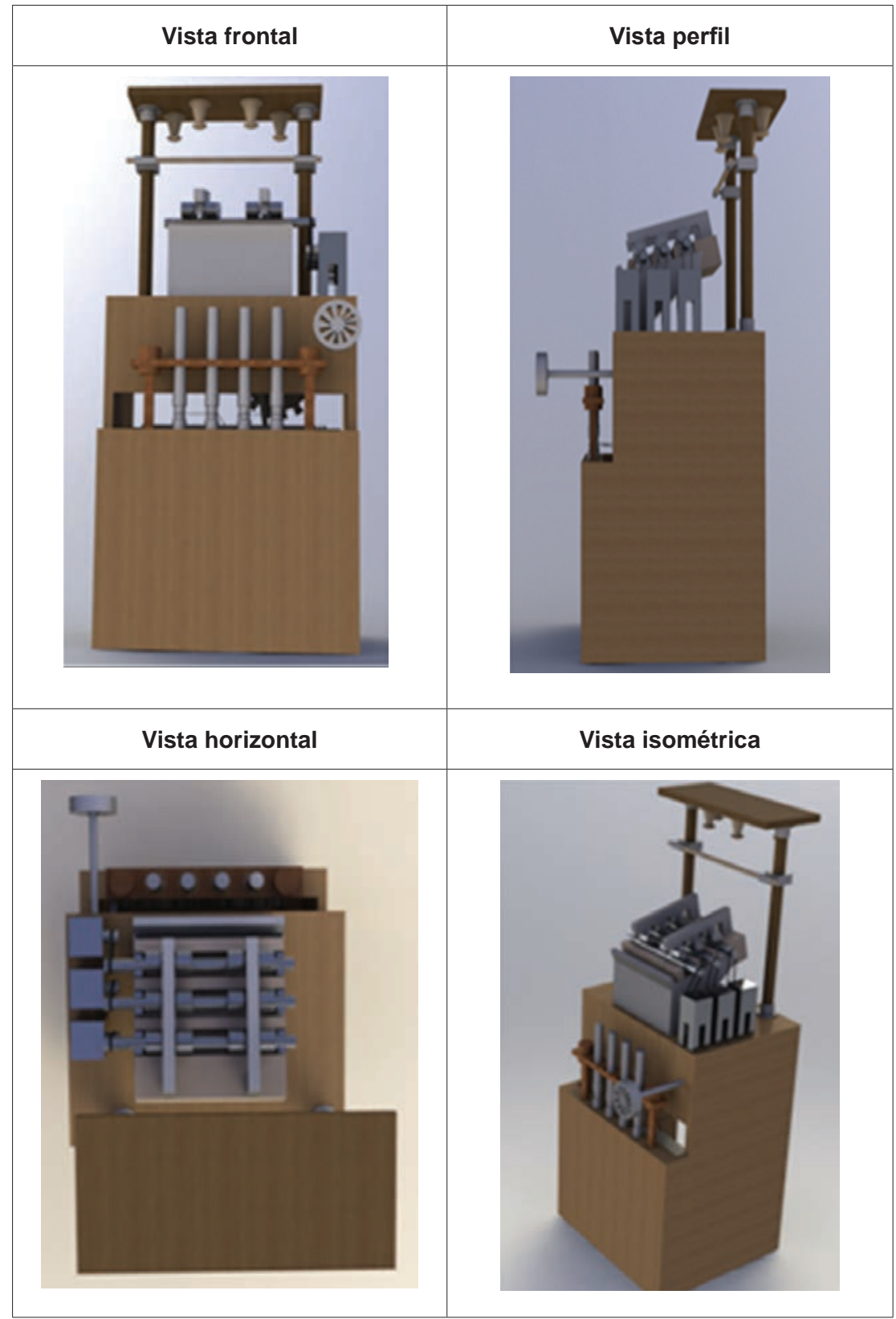




\section{CONCLUSIONES}

Al concluir el estudio, se pueden adelantar los siguientes hallazgos:

- Cuando se ha hilado artesanalmente el algodón nativo de color no ha sido posible obtener títulos finos (12-30 Ne), pero cuando se ha hecho industrialmente, a partir de mezclas con algodón Tangüis, se han obtenido algunas muestras con título $20 \mathrm{Ne}$.

- Se encontró que se mejoran las características físicas del hilo de algodón nativo de color al procesarlo mezclándolo con algodón Tangüis, encontrándose por ajuste de curva un porcentaje óptimo de mezcla en un rango de 60\% y $40 \%$ de ANC y Tangüis, respectivamente.

- Se postula la posibilidad de hilar industrialmente algodón 100\% de ANC mediante el incremento del número de rodillos con que cuenta el tren de estiraje, como el primer paso para darle la torsión de la mecha ${ }^{11}$ y acortar la distancia de encartamiento. ${ }^{12}$

- Las telas tejidas con hilo proveniente de la mezcla 60\% de ANC y 40\% de Tangüis tienen características adecuadas para la costura de prendas de vestir; las características finales de ambas telas dependerán del número de cabos utilizados para el tejido.

- Existe un mercado por satisfacer tanto externo como interno, ya que la tendencia mundial es migrar hacia lo natural y lo orgánico, por ello, es un momento adecuado para la inversión en la producción y procesamiento de este tipo de fibra.

\section{REFERENCIAS}

1. DeTextiles (2009). Control de calidad textil. Recuperado el 17 de noviembre de 2011, de http://www.detextiles.com/files/ CONTROL\%20DE\%20CALIDAD\%20TEXTIL.pdf

2. Hollen, N., Saddler, J. \& Langford, A. (1987). Introducción a los textiles. México D.F.: Limusa.

3. Ingeniería textil (2009). Estiraje. Recuperado 12 de febrero del 2012, de http://opteratextil.blogspot.com/2010/04/estiraje.html

11 Elemento que ingresa a la continua, la cual ha provenido de una mechera.

12 Distancia entre los rodillos del tren de estiraje. 
4. Lazo, J. (1997). Algodones de color: Una alternativa para costa y selva del Perú. Revista para el Desarrollo Agropecuario, Agroindustrial y Agroexportador, 86, 18.

5. Marsal, F. Bader \& Rierola, J. (2002). Experiencias en el nuevo sistema de hilatura por compactación. Madrid: Aitex.

6. Mincetur, Cámara de Comercio de Lima \& Senati (2009). Línea artesanal de tejidos en algodón nativo. Lima: Congreso del Perú, Fondo Editorial.

7. Red Textil Argentina (2008). Hilatura de algodón cardado. Recuperado el 11 de febrero de 2012, de http://www.redtextil argentina.com.ar/index.php/component/content/article/175.html

8. Vreeland, Jr. \& James, M. (1999). The revival of colored cotton. Recuperado el 20 de agosto de 2011, de http://www.perunaturtex. com/scientif.htm

9. Wakelyn, Phillip J. (2010). Producción de hilo de algodón. En Oficina Internacional del Trabajo (Ed.). Enciclopedia de salud y seguridad en el trabajo (pp. 9-12). Ginebra: OIT.

10. Warshaw, León J. (2010). La industria textil: Historia, seguridad y salud. En Oficina Internacional del Trabajo (Ed.). Enciclopedia de salud y seguridad en el trabajo (pp. 2-5). Ginebra: OIT. 